\title{
Desconhecimento de profissionais e ações de extensão quanto à esporotricose no extremo Sul do Brasil
}

\author{
Vanice Rodrigues Poester ${ }^{\mathrm{a}, \mathrm{b}}$, Lisane Almeida Saraiva ${ }^{\mathrm{c}}$, Ana Carolina Pretto ${ }^{\mathrm{a}}$, \\ Gabriel Baracy Klafke ${ }^{\mathrm{a}}$, Karine Ortiz Sanchotene ${ }^{\mathrm{a}}$, Aryse Martins Melo ${ }^{\mathrm{a}}$, \\ Shirlei Cardone ${ }^{\mathrm{d}}$, Melissa Orzechowski Xavier ${ }^{\mathrm{a}, \mathrm{b}, *}$
}

${ }^{a}$ Laboratório de Micologia, Faculdade de Medicina, Universidade Federal do Rio Grande, Rio Grande, RS, Brasil

${ }^{\text {b} P r o g r a m a ~ d e ~ P o ́ s-g r a d u a c ̧ a ̃ o ~ e m ~ C i e ̂ n c i a s ~ d a ~ S a u ́ d e, ~ F a c u l d a d e ~ d e ~ M e d i c i n a, ~ U n i v e r s i d a d e ~ F e d e r a l ~ d o ~ R i o ~ G r a n d e, ~}$ Rio Grande, RS, Brasil

${ }^{\mathrm{c}}$ Prefeitura Municipal de São José do Norte, São José do Norte, RS, Brasil

${ }^{\mathrm{d}}$ Prefeitura Municipal de Rio Grande, Rio Grande, RS, Brasil

Histórico do Artigo

Recebido em:

06/08/2018

Aceito em:

09/10/2018

Palavras-chave:

Epidemiologia;

Sporothrix; Zoonose;

Doenças

Negligenciadas;

Capacitação.

\section{RESUMO}

Atualmente o aumento crescente e alarmante de casos de esporotricose na região Sul do Rio Grande do Sul (RS) acarreta um grave problema para a saúde pública local. Nesta linha, estudos que visem o conhecimento sobre a doença nesta região tornam-se de extrema importância para a compreensão do panorama epidemiológico. Tendo isto em vista, o estudo teve como objetivo avaliar o conhecimento de profissionais da saúde sobre a esporotricose em área hiperendêmica e relatar as atividades de extensão que estão sendo realizadas neste âmbito. Foram aplicados questionários semi-estruturados a profissionais de saúde humana, de dois municípios do sul do RS, totalizando 91 entrevistados. Apesar da alta incidência da doença na região, 26\% dos profissionais não conheciam a doença, e cerca de $40 \%$ apontaram erroneamente ou desconheciam o agente etiológico (38\%) e a principal apresentação clínica da esporotricose (45\%). Considerando a necessidade da suspeição clínica para o diagnóstico e correto tratamento da doença, ações de capacitação foram realizadas abrangendo profissionais da saúde humana e animal. Levando em consideração a alta endemicidade da doença na região, faz-se necessária uma continuidade dessas ações de extensão, com intuito de conscientização dos profissionais e da população quanto ao grave cenário atual da esporotricose, buscando frear o atual aumento do número de casos da doença nesta região.

Professional unfamiliarity and educational intervention about sporotrichosis in a hiperendemic area from Southern Brazil

\section{ABSTRACT}

Currently, the increase in sporotrichosis number cases in the southern region of Rio Grande do Sul (RS) result in a serious problem for local public health. In this line, epidemiological studies, focusing on the knowledge of health professionals, would help to understand the general disease panorama of the region. Taken this into consideration, the study aimed evaluates the knowledge of health professionals about sporotrichosis in a hyperendemic area and to report the extension activities that are being performed in this area. Semi-structured questionnaires were applied in human health professionals from two municipalities in the southern region of RS, totaling 91 respondents. Despite the high incidence of the disease in the region, $26 \%$ of the professionals did not know the disease, and about $40 \%$ did not know the etiological agent $(38 \%)$ and the main clinical presentation of sporotrichosis $(45 \%)$. Considering the need for clinical suspicion for the diagnosis and correct treatment of the disease, training actions were carried out covering human and animal health professionals. Given the high endemicity of the disease in the region, it is necessary to continue these extension actions. Focusing, on raising awareness professionals and population about the current severe scenario of sporotrichosis, seeking to curb the current increase in the number of cases of the disease in this region.

\footnotetext{
*Autor correspondente: melissaxavierfurg@gmail.com ou melissaxavier@ furg.br (Xavier M.O.)
} 


\section{Introdução}

A esporotricose é a micose subcutânea com maior ocorrência no Brasil. Atualmente o número de casos da doença, relacionados com a transmissão zoonótica, têm aumentado em uma proporção alarmante $(1,2)$. Esta transmissão ocorre principalmente pelo envolvimento de gatos domésticos (Feliscatus), devido à forma grave que a doença manifesta-se nestes animais, com lesões disseminadas e alta carga fúngica, somado aos seus hábitos de vida, com arranhaduras e mordeduras comumente descritas (3-6).

No Brasil, os estados com maior número de casos da doença são Rio de Janeiro, São Paulo e Rio Grande do Sul (2,7). No Rio de Janeiro, a esporotricose já é classificada como epidemia urbana, com o relato de 5.000 casos de esporotricose zoonótica nos últimos dezessete anos, sendo que atualmente a doença têm se dispersado geograficamente para outros municípios próximos ao epicentro da doença $(2,3,8)$.

O segundo estado com maior incidência da doença é o Rio Grande do Sul (RS), sendo que os casos concentram-se na região sul (6, 9-11). Esta região é hiperendêmica para a esporotricose, sendo descritos casos desde a década de 90 e, mais recentemente um agravamento do cenário regional, com centenas de casos diagnosticados em felinos e dezenas em humanos no período de sete anos (2010-2016) (6, 9-13). Tendo em vista, que o aumento de casos da doença nestes animais precede o aumento de casos em humanos, trazendo um grave problema de saúde pública, estudos epidemiológicos são de extrema relevância para o entendimento da situação regional (3-6).

Nesta linha, a realização de estudos que visam avaliar o conhecimento dos profissionais da área da saúde sobre a doença pode auxiliar na avaliação do panorama epidemiológico. Considerando que o papel dos profissionais da saúde como multiplicadores de informações corretas é essencial para auxiliar no controle e prevenção da esporotricose tanto em animais como em humanos, a avaliação do conhecimento destes profissionais acerca da doença torna-se relevante para a compreensão da problemática e adoção de medidas em áreas hiperendêmicas. Com isso, o objetivo do estudo foi avaliar o conhecimento de profissionais da saúde de dois municípios da região sul do Rio Grande do Sul, Brasil, sobre a esporotricose, e realizar ações de extensão no intuito de facilitar o acesso ao diagnóstico da doença e contribuir para o empoderamento dos profissionais e da população no âmbito deste conhecimento.

\section{Materiais e métodos}

Foram incluídos no estudo profissionais da saúde que trabalham na Rede de Atenção Básica e Clínicas Particulares de dois municípios da região sul do RS, sendo o município 1 com uma população de cerca de 250mil habitantes e o município $2 \mathrm{com}$ cerca de $25 \mathrm{mil}$ habitantes (14). Os dados foram coletados a partir de questionário semiestruturado com perguntas referentes ao conhecimento em geral sobre a esporotricose, abordando agente etiológico, forma de transmissão, apresentação clínica, ocorrência na região, entre outras.

No município 1 a coleta de dados ocorreu em julho de 2017, previamente a um evento de capacitação sobre a doença e sua importância na região. Neste evento participaram médicos $(n=29)$ e enfermeiros $(n=25)$ atuantes em 20 distintas Unidades Básicas de Saúde da Família do município e todos responderam ao questionário ao ingressar no evento. No município 2, a coleta de dados ocorreu em setembro de 2017, com busca ativa de profissionais da saúde, a partir da visitação das Unidades de Saúde do município. Todos os participantes incluídos no estudo, não haviam participado de nenhum evento de capacitação disponibilizado anteriormente, estes entrevistados foram capacitados após o preenchimento do questionário. Foram incluídos no estudo todos os 
profissionais que concordaram em participar da pesquisa, assinando o Termo de Consentimento Livre e Esclarecido (TCLE). O estudo foi aprovado pelo Comitê de Ética em Pesquisa na Área da Saúde (CEPAS) da Universidade Federal do Rio Grande - FURG, sob o parecer 146/2016.

Quanto às ações de extensão, três eventos de capacitação foram realizados, no município 1, pelo Laboratório de Micologia da Faculdade de Medicina da FURG, nos anos de 2011, 2013 e 2017, com palestras e debates sobre a esporotricose felina e zoonótica na região. O primeiro contou com a parceria da Vigilância Ambiental em Saúde, e o terceiro da Vigilância Epidemiológica da Secretaria de Saúde, ambos pertencentes ao município 1 .

Durante estes eventos foi proposto e disponibilizado aos participantes, apoio do Laboratório de Micologia (LM) para realização de exames micológicos para diagnóstico da enfermidade em animais e humanos, mediante cadastro, com discussão e definição da melhor logística para encaminhamento de pacientes e/ou amostras. Além disso, nos eventos foram explanadas informações gerais sobre a ocorrência e prevalência de casos de esporotricose zoonótica e animal, tendo como base estudos sobre a epidemia da doença no estado do Rio de Janeiro, com intuito do fornecimento de dados sobre a crescente importância desta doença para a saúde pública $(2,3,8)$. Adicionalmente, quando disponível, foram apresentados dados sobre a ocorrência de casos da doença, em animais e humanos, na região de estudo $(6,12)$.

Nos eventos de 2011 e 2013 a maior parte dos participantes eram profissionais da saúde animal, já no evento de 2017 somente participaram profissionais da saúde humana. Apesar de os eventos terem ocorrido apenas no município 1, todos os profissionais entrevistados do município 2 receberam material informativo e todos questionamentos foram esclarecidos para estes entrevistados ao final da coleta de dados.

As respostas dos participantes foram avaliadas pela análise de frequência, utilizando o programa estatístico SPSS 20.0.

\section{Resultados}

Durante o período do estudo, foram convidados a participar da pesquisa 95 profissionais da saúde, havendo recusa em quatro casos, totalizando 91 profissionais incluídos no estudo. Destes, 44\% (40/91) eram enfermeiros, 40\% (36/91) médicos, 15\% (14/91) técnicos em enfermagem, e 1\% (1/91) bioquímicos. Dentre estes, a maior parte atuava na saúde pública, correspondendo a 96\% (87/91) dos entrevistados.

Apenas metade dos profissionais respondeu ter conhecimento da ocorrência de casos de esporotricose nos municípios. Em adição, cerca de $40 \%$ destes não sabia ou apontou erroneamente o agente etiológico $(38 \% ; 33 / 86)$ e a principal apresentação clínica da doença $(45 \%$; 38/87). Quanto à forma de transmissão, somente 30\% dos entrevistados respondeu corretamente haver necessidade de inoculação traumática para desencadeamento da doença, e 32\% (28/88) desconheciam a importância do felino doméstico como transmissor da doença para humanos e outros animais (Tabela 1). 
Tabela 1- Respostas dos participantes, conforme área de atuação, sobre a esporotricose no Sul do Rio Grande do Sul (n=91).

\begin{tabular}{|c|c|c|c|c|}
\hline \multicolumn{2}{|c|}{ Respostas } & \multirow{2}{*}{$\frac{\text { Medicina }}{94 \%(34 / 36)}$} & \multirow{2}{*}{$\begin{array}{r}\text { Enfermagem } \\
60 \%(31 / 52)\end{array}$} & \multirow{2}{*}{$\begin{array}{l}\text { Total* } \\
74 \%(65 / 88)\end{array}$} \\
\hline Conhece & Sim & & & \\
\hline & Não & $6 \%(2 / 36)$ & $40 \%(21 / 52)$ & $26 \%(23 / 88)$ \\
\hline \multirow[t]{3}{*}{ Etiologia } & Correto & $80 \%(29 / 36)$ & $48 \%(24 / 50)$ & $62 \%(53 / 86)$ \\
\hline & Incorreto & $15 \%(5 / 36)$ & $20 \%(10 / 50)$ & $17 \%(15 / 86)$ \\
\hline & Desconhece & $5 \%(2 / 36)$ & $32 \%(16 / 50)$ & $21 \%(18 / 86)$ \\
\hline Apresentação & Correto & $61 \%(22 / 36)$ & $51 \%(26 / 51)$ & $55 \%(48 / 87)$ \\
\hline \multirow[t]{2}{*}{ Clínica } & Incorreto & $36 \%(13 / 36)$ & $14 \%(7 / 51)$ & $23 \%(20 / 87)$ \\
\hline & Desconhece & $3 \%(1 / 36)$ & $35 \%(18 / 51)$ & $22 \%(19 / 87)$ \\
\hline \multirow[t]{3}{*}{ Transmissão } & Correto & $43 \%(15 / 35)$ & $22 \%(11 / 51)$ & $30 \%(26 / 87)$ \\
\hline & Incorreto & $57 \%(20 / 35)$ & $43 \%(22 / 51)$ & $49 \%(42 / 86)$ \\
\hline & Desconhece & $0 \%(0 / 35)$ & $35 \%(18 / 51)$ & $21 \%(18 / 86)$ \\
\hline \multirow[t]{3}{*}{ Animal Envolvido } & Gato & $70 \%(25 / 36)$ & $67 \%(35 / 52)$ & $68 \%(60 / 88)$ \\
\hline & Outros & $22 \%(8 / 36)$ & $10 \%(5 / 52)$ & $15 \%(13 / 88)$ \\
\hline & Desconhece & $8 \%(3 / 36)$ & $23 \%(12 / 52)$ & $17 \%(15 / 88)$ \\
\hline
\end{tabular}

*n total de cada variável corresponde ao número de participantes que respondeu ao questionamento, sendo os demais (n faltante para completar 91) considerado "missing" de cada variável.

Após a aplicação do questionário, todos os participantes receberam material ilustrativo e informativo (Figura 1) sobre a doença, os quais foram entregues para uso individual do entrevistado, bem como para divulgação e disponibilização nos estabelecimentos de saúde com o intuito de transmitir a informação para a população em geral, contribuindo para o empoderamento da população usuária dos serviços.

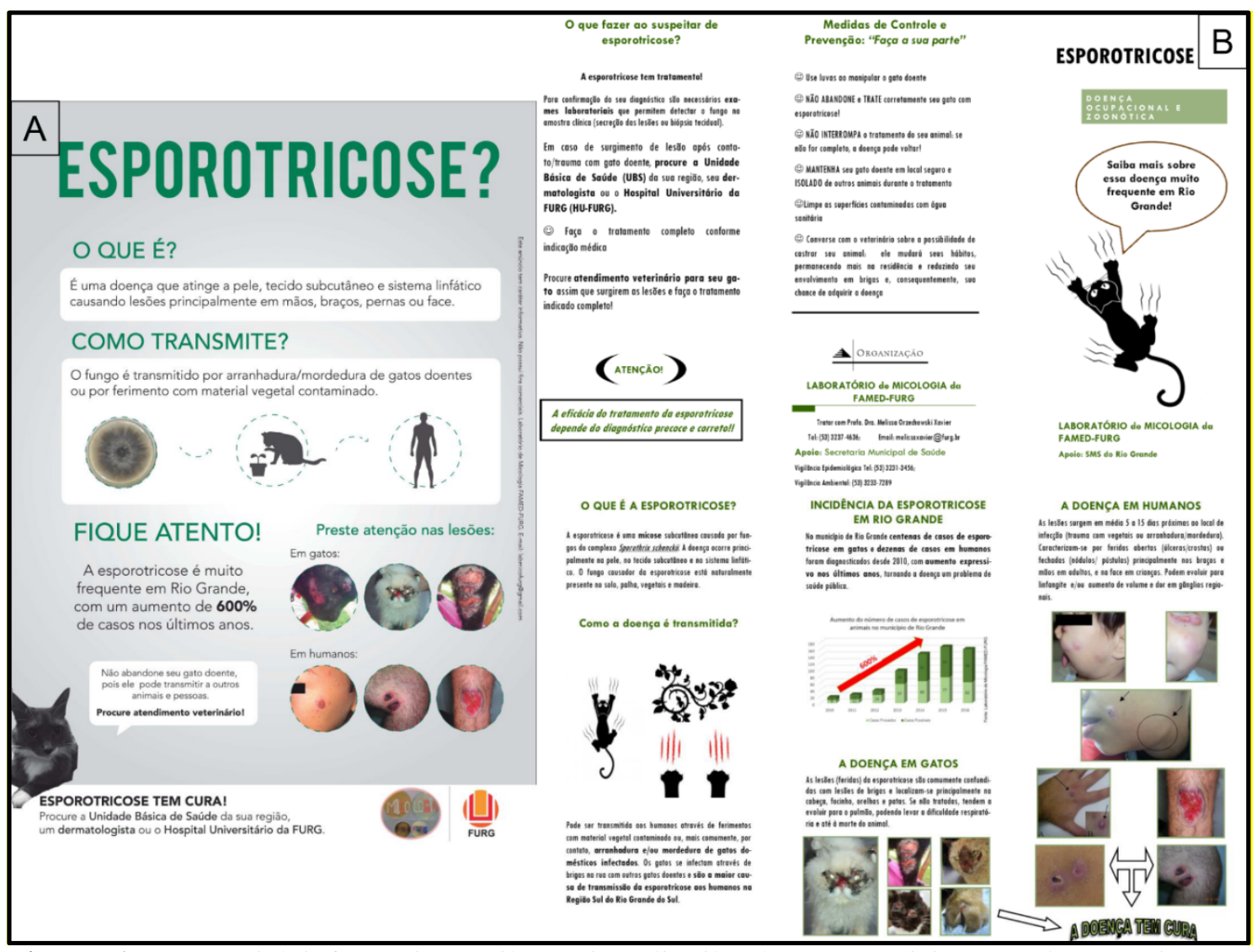

Figura 1 - Material informativo e ilustrativo distribuído aos participantes da pesquisa: A. Cartaz (420x297mm); B. Folder (frente e verso). 
Quanto aos eventos de extensão, ao todo foram 144 profissionais da área da saúde capacitados, sendo 42 médicos, 27 enfermeiros, 51 médicos veterinários e 24 outros (farmacêuticos, bioquímicos, biomédicos, biólogos), e 15 estudantes de graduação ou pós-graduação. Após estes eventos, o LM tem atualmente 40 veterinários cadastrados que aderiram ao projeto tendo acesso ao apoio laboratorial para diagnóstico micológico da esporotricose felina de forma gratuita. Da mesma forma, foi organizada uma logística junto a SMS do município 1 em que todo paciente humano com suspeita de esporotricose atendido na Rede Básica de Saúde é encaminhado ao LM, passando por exame clínico em ambulatório do Hospital Universitário e coleta de amostra para processamento laboratorial e confirmação diagnóstica da doença. Após diagnóstico o paciente retorna ao ambulatório para tratamento e acompanhamento da evolução clínica.

A partir dessas ações, que culminaram com uma centralização do diagnóstico da doença, entre os anos de 2010 e 2017, centenas de casos de esporotricose felina e cerca de 100 casos da doença em humanos, a maioria deles com envolvimento zoonótico, já foram diagnosticados no LM $(6,13)$. E, a partir destes dados de prevalência da doença $(6,13)$, demonstrados via projeto do LM-FAMED-FURG, foi emitida pela Vigilância Epidemiológica a Circular $\mathrm{n}^{\circ} 005 / 2017$ de 31 de julho de 2017, determinando o fornecimento gratuito pela SMS do antifúngico (itraconazol) para tratamento da esporotricose em indivíduos com diagnóstico comprovado. Atualmente, todos os casos em humanos diagnosticados pelo LM-FAMED-FURG estão sendo notificados junto a Vigilância Epidemiológica do município 1.

\section{Discussão}

O Rio Grande do Sul é o segundo estado com maior ocorrência de esporotricose no Brasil, neste local os casos estão concentrados na Região Sul $(2,6,9-12,15,16)$, apesar disto, 26\% dos entrevistados não conheciam a doença. Da mesma forma, cerca de $90 \%$ dos casos de esporotricose em humanos tem acometimento cutâneo e/ou subcutâneo $(3,11)$, após inoculação traumática do fungo (17). Estes dados foram apontados erroneamente ou eram desconhecidos por $45 \%$ e $70 \%$, respectivamente, dos profissionais entrevistados. Tendo em vista, que lesões de esporotricose não são específicas da doença, podendo ser erroneamente relacionadas a outras enfermidades, é necessário que os profissionais de saúde estejam cientes da alta endemicidade da doença na região e familiarizados com a principal apresentação clínica da doença, seus sinais e sintomas,e via de infecção, aumentando assim sua suspeição para que haja a confirmação diagnóstica $(17,18)$.

O conhecimento sobre a importância do felino doméstico na disseminação da esporotricose intra e interespécie, sendo a principal fonte de infecção do fungo S. brasiliensis para humanos e outros animais (2) é essencial para que medidas corretas de prevenção e controle sejam adotadas por pessoas que tenham esses animais como pets ou que trabalhem com os mesmos (17), buscando reduzir os casos zoonóticos. No entanto, 38\% dos profissionais entrevistados desconhecia o envolvimento dos felinos na transmissão da doença, apesar de estarem atuando em uma região de alta endemicidade de esporotricose felina $(6,13)$.

Embora as ações propostas já estejam gerando benefícios a população, como maior acessibilidade ao diagnóstico e gratuidade no tratamento da doença em humanos, nosso estudo demonstrou que ainda há déficit de conhecimento da área da saúde quanto a esporotricose, gerando agravantes para a progressão da hiperendemia regional. De fato, a esporotricose é considerada uma doença negligenciada, sendo esta uma problemática de cunho nacional (19), e que culminou com a sua epidemia no estado do RJ, onde desde a década de 90 a literatura evidenciava a necessidade de adoção de medidas de 
ações públicas de controle e prevenção $(19,20)$, e devido a negligencia e a pouca atenção voltada a esta problemática, o estado chegou a uma situação epidêmica, com mais de 5000 casos descritos apenas em humanos (2).

\section{Conclusão}

Considerando que a esporotricose é uma doença cuja transmissão pode ocorrer tanto pelo ambiente, como por felinos infectados, a sua problemática para a saúde pública tem que ser tratada como um conjunto indissociável para esta tríade (ambiente-felino-humano), conceituado como saúde única $(2,17,21)$. Nesta linha, ações públicas para controle desta doença têm que atuar de forma multidisciplinar, visando principalmente: - controle da esporotricose felina em animais errantes, que são grande foco de transmissão para outros animais e até humanos e não recebem a devida atenção quanto a tratamento e quanto a cuidados básicos; - conscientização dos proprietários de felinos quanto à posse responsável e a importância do cuidando correto do seu animal, evitando que este tenha livre acesso a rua, e de medidas que diminuam o risco de transmissão da doença, como a castração; conscientização dos profissionais de saúde humana e animal e da população em geral, para que a esporotricose seja cotada como uma doença de alta ocorrência na região, aumentando assim a sua suspeição clínica $(6,13,17,20)$. Com isso, há a necessidade de seguir de forma continuada com as estas ações educativas e de interação Universidade/Comunidade buscando melhorias na prevenção e controle da esporotricose com intuito de reduzir sua alta prevalência na região.

\section{Agradecimentos}

Os autores são gratos a Coordenação de Aperfeiçoamento de Pessoal de Nivel Superior (CAPES) pela concessão de bolsas de estudo.

\section{Referências}

1. Mackinnon JE, Conti-Diaz IA, Gezuela E, Civila E, Luz S. Isolation of Sporothrix schenckii from nature considerations on its pathogenicity and ecology. Sabouraudia 1969; 7: 38-45.

2. Gremião IDF, Miranda LHM, Reis EG, Rodrigues AM, Pereira SA. Zoonotic Epidemic of Sporotrichosis : Cat to Human Transmission. PLOS Pathog 2017;13(1):e1006077.

3. Barros MBL, Schubach AO, Schubach TMP, Wanke B, Lambert-Passos SR. An epidemic of sporotrichosis in Rio de Janeiro, Brazil: epidemiological aspects of a series of cases. Epidemiol Infect 2008;136(9):1192-6.

4. Montenegro H, Rodrigues AM, Dias MAG, da Silva EA, Bernardi F, de Camargo ZP. Feline sporotrichosis due to Sporothrix brasiliensis: an emerging animal infection in São Paulo, Brazil. BMC Vet Res 2014;10: 269.

5. Gremião ID, Menezes RC, Schubach TM, Figueiredo AB, Cavalcanti MC, Pereira SA. Feline sporotrichosis: epidemiological and clinical aspects. Med Mycol. 2015;53:15-21.

6. Sanchotene KO, Madrid IM, Klafke GB, Bergamashi M, Terra PPD, Rodrigues AM, et al. Sporothrix brasiliensis outbreaks and the rapid emergence of feline sporotrichosis. Mycoses 2015;58(11):652-8.

7. Gutierrez-Galhardo MC, Freitas DFS, do Valle ACF, Almeida-Paes R, de Oliveira MME, Zancopé-Oliveira RM. Epidemiological Aspects of Sporotrichosis Epidemic in Brazil. Curr Fungal Infect Rep 2015;9(4):238-45.

8. Almeida P, Inês M, Giordano C. Vigilância e cenário epidemiológico: Esporotricose no estado do RJ. Bol Epidemiológico Esporotricose007/2016; 2016.

9. Nobre MO, Potter-de-Castro A, Caetano D, Leonardo-de-Souza, L Araujo-Meireles, MC Ferreiro L. Recurrenceofsporotrichosis in cats with zoonotic involvement. Ver Iberoam Micol 2001; 18:137-40. 
10. Xavier MO, Nobre MO, Sampaio Junior DP, Antunes TA, Nascente PS, Sória FAB, et al. Esporotricose felina com envolvimento humano na cidade de Pelotas, RS, Brasil. Ciência Rural 2004;34:1961-3.

11. Xavier MO, Bittencourt LR, Silva CM, Vieira RS, Pereira HC. Atypical presentation of sporotrichosis: report of three cases. Rev Soc Bras Med Trop 2013; 46:116-8.

12. Madrid IM, Mattei AS, Fernandes CG, Oliveira Nobre M, Meireles MCA. Epidemiological findings and laboratory evaluation of sporotrichosis: a description of 103 cases in cats and dogs in Southern Brazil. Mycopathologia 2012;173:265-73.

13. Poester VR, Mattei AS, Madrid IM, Pereira JTB, Klafke GB, Sanchotene KO, et al. Sporotrichosis in Southern Brazil, towards an epidemic? Zoonoses and Public Health 2018; 65(7):815-821.

14. IBGE Instituto Brasileiro de Geografia e Estatística. Censo Demográfico 2010. 2016. Availablefrom: http://cidades.ibge.gov.br/xtras/perfil.php?codmun=431560

15. Lopes JO, Alves SH, Mari CR, Brum LM, Westphalen JB, Altermann MJ, et al. Epidemiologia da esporotricosena região central do Rio Grande do Sul. Rev Soc Bras Med Trop 1999; 32(5): 541-5.

16. Rosa ACM da, Scroferneker ML, Vettorato R, Gervini RL, Vettorato G, Weber A. Epidemiology of sporotrichosis: A study of 304 cases in Brazil. An Acad Dermatology 2005;52(3):451-9.

17. Barros MBDL, de Almeida Paes R, Schubach AO. Sporothrix schenckii and Sporotrichosis. Clin Microbiol Rev2011;24(4):633-54.

18. Peter JR, Silva R, Andrade FC. A esporotricose e seu impacto social. Vittalle 2016;28:111-4.

19. Silva MBT, Costa MM de M, Torres CC da S, Galhardo MCG, do Valle ACF, Magalhães M de AFM, et al. Esporotricose urbana: Epidemia negligenciada no Rio de Janeiro, Brasil. Cad Saúde Pública 2012;28(10):1867-80.

20. Barros MBL, Schubach TMP, Coll JO, Gremião ID, Wanke B, Schubach AO. Esporotricose: a evolução e os desafios de uma epidemia. Rev Panam Salud Pública 2010; 27:455-60.

21. Centers for Disease Control and Prevention C. One Health basics.2016. 